\title{
PRESENTATIONS FOR SOME MONOIDS OF PARTIAL TRANSFORMATIONS ON A FINITE CHAIN"
}

\section{Vítor H. Fernandes}

Centro de Álgebra da Universidade de Lisboa, Lisboa, Portugal; and Departamento de Matemática, Universidade Nova de Lisboa, Caparica, Portugal

\section{Gracinda M. S. Gomes}

Centro de Álgebra da Universidade de Lisboa, Lisboa, Portugal; and Departamento de Matemática, Universidade de Lisboa, Lisboa, Portugal

\section{Manuel M. Jesus}

Centro de Álgebra da Universidade de Lisboa, Lisboa, Portugal; and Departamento de Matemática, Universidade Nova de Lisboa, Caparica, Portugal

In this paper we calculate presentations for some natural monoids of transformations on a chain $X_{n}=\{1<2<\cdots<n\}$. First we consider $\mathscr{O} \mathscr{D}_{n}\left[\mathscr{P} \mathscr{O} \mathscr{D}_{n}\right.$ ], the monoid of all full [partial] transformations on $X_{n}$ that preserve or reverse the order. Two other monoids of partial transformations on $X_{n}$ we look at are $\mathscr{P} \mathscr{O P} \mathscr{P}_{n}$ and $\mathscr{P} \mathscr{O} \mathscr{R}_{n}$-the elements of the first preserve the orientation and the elements of the second preserve or reverse the orientation.

Key Words: Monoid; Order-preserving; Orientation-preserving; Presentation; Transformation.

2000 Mathematics Subject Classification: 20M20; 20M05; $20 \mathrm{M} 18$.

\section{INTRODUCTION AND PRELIMINARIES}

Semigroups of order-preserving transformations have long been considered in the literature. Arzenštat (1962) and Popova (1962) exhibited presentations for $\sigma_{n}$, the monoid of all order-preserving full transformations on a chain with $n$ elements, and for $\mathscr{P} \mathscr{O}_{n}$, the monoid of all order-preserving partial transformations on a chain with $n$ elements. Some years later, Howie (1971) studied some combinatorial and algebraic properties of $\mathscr{O}_{n}$ and the second author together with Howie (1992) revisited the monoids $\mathscr{O}_{n}$ and $\mathscr{P} \mathscr{O}_{n}$. More recently, the injective counterpart of $\mathscr{O}_{n}$, i.e., the monoid $\mathscr{P} \mathscr{G} \mathscr{F}_{n}$ of all injective members of $\mathscr{P} \mathscr{O}_{n}$, has been the object of study

Received July 2003; Revised January 2005

\#Communicated by P. Higgins.

Address correspondence to Gracinda M. S. Gomes, Centro de Álgebra da Universidade de Lisboa, Av. Prof. Gama Pinto, 2, 1649-003 Lisboa, Portugal; E-mail: ggomes@cii.fc.ul.pt 
by the first author in several papers $(1997,1998,2001,2002 a, 2002 b)$ and also by Cowan and Reilly (1995).

On the other hand, the notion of an orientation-preserving transformation was introduced by McAlister (1998) and, independently, by Catarino and Higgins (1999). The monoid $\mathscr{G P}_{n}$, of all orientation-preserving full transformations on a chain with $n$ elements, was also considered by Catarino (1998) and by Arthur and Ruškuc (2000). The injective counterpart of $\mathscr{O} \mathscr{P}_{n}$, i.e., the monoid $\mathscr{P} \mathscr{C P} \mathscr{P}_{n}$ of all injective orientation-preserving partial transformations on a chain with $n$ elements, was studied by the first author $(2000,2001)$.

Recently the authors exhibited presentations for the monoids $\mathscr{P} \mathscr{D} \mathscr{D} \mathscr{F}_{n}$ of all injective order-preserving or order-reversing partial transformations on a chain with $n$ elements, and for the monoid $\mathscr{P} \mathscr{R} \mathscr{S}_{n}$ of all injective orientation-preserving or orientation-reversing partial transformations on a chain with $n$ elements (Fernandes et al., to appear).

Delgado and Fernandes (2000) have computed the abelian kernels of the monoids $\mathscr{P G} \mathscr{F}_{n}$ and $\mathscr{P} \mathscr{C P} \mathscr{F}_{n}$, using a method that is strongly dependent on the known presentations of these monoids (Fernandes, 2001, 2000). More recently, the same authors Delgado and Fernandes (2004) also calculated the abelian kernels of the monoids $\mathscr{P} \mathscr{C D} \mathscr{F}_{n}$ and $\mathscr{P} \mathscr{O} \mathscr{R} \mathscr{F}_{n}$. Again, the knowledge of the presentations played a crucial role.

In this paper, we give presentations for the monoid $\mathscr{O} \mathscr{D}_{n}$ of all order-preserving or order-reversing full transformations on a chain with $n$ elements, in terms of $n$ generators and $\left(n^{2}+n+2\right) / 2$ relations; for the monoid $\mathscr{P} \mathscr{O} \mathscr{D}_{n}$ of all orderpreserving or order-reversing partial transformations on a chain with $n$ elements, in terms of $[n / 2\rceil+n$ generators and $\left\{7 n^{2}+2 n+(3 / 2)\left[1-(-1)^{n}\right]\right\} / 4$ relations; for the monoid $\mathscr{P} \mathscr{G} \mathscr{P}_{n}$ of all orientation-preserving partial transformations on a chain with $n$ elements, in terms of three generators and $4 n+2$ relations; and, finally, for the monoid $\mathscr{P} \mathscr{O} \mathscr{R}_{n}$ of all orientation-preserving or orientation-reversing partial transformations on a chain with $n$ elements, in terms of four generators and $4 n+7$ relations.

We would like to point out that to guess some of the presentations we made considerable use of computational tools: namely, we used McAlister's program Semigroup for Windows (1997) and GAP (2002).

Next, we will introduce some definitions.

Let $X$ be a set. As usual, we denote by $\mathscr{P} \mathscr{T}(X)[\mathscr{T}(X)]$ the monoid of all partial [full] transformations of $X$.

Let $X_{n}$ be a chain with $n$ elements, say $X_{n}=\{1<2<\cdots<n\}$.

We say that a transformation $s$ in $\mathscr{P} \mathscr{T}\left(X_{n}\right)$ is order-preserving [order-reversing] if, for all $x, y \in \operatorname{Dom}(s), x \leq y$ implies $x s \leq y s[x s \geq y s]$. Clearly, the product of two order-preserving transformations or of two order-reversing transformations is orderpreserving and the product of an order-preserving transformation by an orderreversing transformation is order-reversing.

We denote by $\mathscr{P} \mathscr{O}_{n}$ the submonoid of $\mathscr{P} \mathscr{T}\left(X_{n}\right)$ whose elements are orderpreserving and by $\mathscr{P} \mathscr{O} \mathscr{D}_{n}$ the submonoid of $\mathscr{P} \mathscr{T}\left(X_{n}\right)$ whose elements are either order-preserving or order-reversing. Also, we denote by $\mathscr{O}_{n}\left[\mathscr{O O D}_{n}\right]$ the submonoid of $\mathscr{P} \mathscr{O}_{n}\left[\mathscr{P} \mathscr{O} \mathscr{D}_{n}\right]$ whose elements are full transformations.

Now, let $a=\left(a_{1}, a_{2}, \ldots, a_{t}\right)$ be a sequence of $t(t \geq 0)$ elements from the chain $X_{n}$. We say that $a$ is cyclic [anti-cyclic] if there exists no more than one index $i \in$ $\{1, \ldots, t\}$ such that $a_{i}>a_{i+1}\left[a_{i}<a_{i+1}\right]$, where $a_{t+1}$ denotes $a_{1}$. Let $s \in \mathscr{P} \mathscr{F}\left(X_{n}\right)$ and 


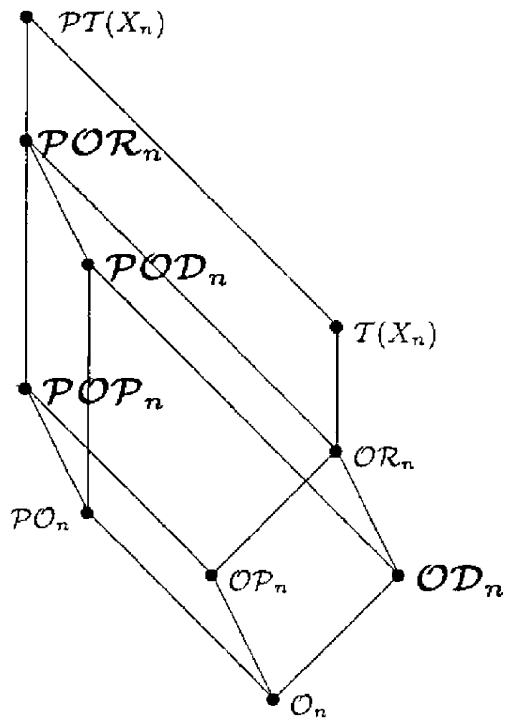

suppose that $\operatorname{Dom}(s)=\left\{a_{1}, \ldots, a_{t}\right\}$, with $t \geq 0$ and $a_{1}<\cdots<a_{t}$. We say that $s$ is an orientation-preserving [orientation-reversing] transformation if the sequence of its images $\left(a_{1} s, \ldots, a_{t} s\right)$ is cyclic [anti-cyclic]. The product of two orientation-preserving or of two orientation-reversing transformations is orientation-preserving and the product of an orientation-preserving transformation by an orientation-reversing transformation is orientation-reversing. We denote by $\mathscr{P} \mathscr{G P}{ }_{n}\left[\mathscr{P} \mathscr{G} \mathscr{R}_{n}\right]$ the monoid of all orientation-preserving [preserving or reversing] partial transformations and by $\mathscr{O O P}_{n}[\mathscr{O} \mathscr{R} n]$ we denote the corresponding submonoids of full transformations. The following diagram, with respect to the inclusion relation clarifies the relationship between these various semigroups.

Now, denote by $X^{*}$ the free monoid generated by $X$. A monoid presentation is an ordered pair $\langle X \mid R\rangle$, where $X$ is an alphabet and $R$ is a subset of $X^{*} \times X^{*}$. A monoid $M$ is said to be defined by a presentation $\langle X \mid R\rangle$ if $M$ is isomorphic to $X^{*} / \rho_{R}$, where $\rho_{R}$ denotes the smallest congruence on $X^{*}$ containing $R$. An element $(u, v)$ of $X^{*} \times X$ is called a relation and it is usually represented by $u=v$. We say that $u=v$ is a consequence of $R$ if $(u, v) \in \rho_{R}$. For more details see Lallement (1979) or Ruškuc (1995).

Given a finite monoid $T$, it is clear that we can always exhibit a presentation for it, at worst by enumerating all its elements, but clearly this is of no interest, in general. So, by finding a presentation for a finite monoid, we mean to find in some sense a nice presentation (e.g., with a small number of generators and relations).

To find some presentations we will use the Guess and Prove Method described by the following theorem adapted from Ruškuc (1995, Proposition 3.2.2.).

Theorem 1.1. Let $M$ be a finite monoid. Let $X$ be a generating set for $M$. Let $R \subseteq$ $X^{*} \times X^{*}$ a set of relations and $W \subseteq X^{*}$. that the following conditions are satisfied:

1. The generating set $X$ of $M$ satisfies all the relations from $R$; 
2. For each word $w \in X^{*}$, there exists a word $w^{\prime} \in W$ such that the relation $w=w^{\prime}$ is a consequence of $R$;

3. $|W| \leq|M|$.

Then $M$ is defined by the presentation $\langle X \mid R\rangle$.

Notice that, if $W$ satisfies the above conditions then, in fact, $|W|=|\mathrm{M}|$.

Let $X$ be an alphabet, $R \subseteq X^{*} \times X^{*}$ a set of relations and $W$ a subset of $X^{*}$. We say that $W$ is a set of forms for the monoid defined by the presentation $\langle X \mid R\rangle$ if $W$ is a transversal of $\rho_{R}$.

Given a presentation for a monoid, another method of finding a new presentation consists in applying Tietze transformations. For a monoid presentation $\langle A \mid R\rangle$, we define the four elementary Tietze transformations:

(T1) Adding a new relation $u=v$ to $\langle A \mid R\rangle$, providing that $u=v$ is a consequence of $R$;

(T2) Deleting a relation $u=v$ from $\langle A \mid R\rangle$, providing that $u=v$ is a consequence of $R \backslash\{u=v\}$;

(T3) Adding a new generating symbol $b$ and a new relation $b=w$, where $w \in A^{*}$;

(T4) If $\langle A \mid R\rangle$ possesses a relation of the form $b=w$, where $b \in A$, and $w \in$ $(A \backslash\{b\})^{*}$, then deleting $b$ from the list of generating symbols, deleting the relation $b=w$, and replacing all remaining appearances of by $w$.

The following result is well-known:

Theorem 1.2 (Ruškuc, 1995). Two finite presentations define the same monoid if and only if one can be obtained from the other by a finite number of elementary Tietze transformations (T1), (T2), (T3), and (T4).

Next, we recall a method, due to Fernandes et al. (to appear), of obtaining a presentation for a finite monoid $M$ given a presentation for a certain submonoid of $M$.

Let $M$ be a finite monoid, $S$ a submonoid of $M$ and $y$ an element of $M$ such that $y^{2}=1$. Suppose that $M$ is generated by $S$ and $y$. Let $X=\left\{x_{1}, \ldots, x_{k}\right\}(k \in \mathbb{N})$ be a generating set of $S$ and $\langle X \mid R\rangle$ a presentation for $S$. Consider a set of forms $W$ for $\langle X \mid R\rangle$ and assume there exist subsets $W_{\alpha}$ and $W_{\beta}$ of $W$ and a word $u_{0} \in X^{*}$ such that $W=W_{\alpha} \cup W_{\beta}$ and $u_{0}$ is a factor of each word in $W_{\alpha}$. Let $Y=X \cup\{y\}$. Notice that $Y$ generates $M$. Suppose now that there exist words $v_{0}, v_{1}, \ldots, v_{k} \in X^{*}$ such that the following relations over the alphabet $Y$ are satisfied by the generating set $Y$ of $M$ :

$\left(N R_{1}\right) y x_{i}=v_{i} y$, for all $i \in\{1, \ldots, k\}$;

$\left(N R_{2}\right) u_{0} y=v_{0}$.

Observe that the relation (over the alphabet $Y$ )

$\left(N R_{0}\right) y^{2}=1$

is also satisfied (by the generating set $Y$ of $M$ ), by hypothesis.

Let $\bar{R}=R \cup N R_{0} \cup N R_{1} \cup N R_{2}$ and $\bar{W}=W \cup\left\{w y \mid w \in W_{\beta}\right\} \subseteq Y^{*}$. 
Theorem 1.3 (Fernandes et al., to appear). If $W$ contains the empty word, then $\bar{W}$ is a set of forms for $\langle Y \mid \bar{R}\rangle$. Moreover, if $|\bar{W}| \leq|M|$, then the monoid $M$ is defined by the presentation $\langle Y \mid \bar{R}\rangle$.

\section{A PRESENTATION FOR $\mathscr{O} \mathscr{D}_{n}$}

This section is dedicated to finding two presentations for the monoid $\mathscr{C O D}_{n}$ of all order-preserving or order-reversing full transformations on $X_{n}$. The first will be obtained applying Theorem 1.3 to the Aizenštat (1962) presentation $\langle X \mid A\rangle$ for the submonoid $\mathscr{C}_{n}$, of all order-preserving full transformations. The presentation $\langle X \mid A\rangle$ is given in terms of the $2 n-2$ elements generating set $X=$ $\left\{u_{1}, \ldots, u_{n-1}, v_{1}, \ldots, v_{n-1}\right\}$, where

$$
u_{i}=\left(\begin{array}{ccc|c|ccc}
1 & \cdots & i-1 \\
1 & \cdots & i-1 & i+1 & i+1 & \cdots & n \\
i+1 & \cdots & n
\end{array}\right)
$$

and

$$
v_{i}=\left(\begin{array}{ccc|c|ccc}
1 & \cdots & n-i & n-i+1 & n-i+2 & \cdots & n \\
1 & \cdots & n-i & n-i & n-i+2 & \cdots & n
\end{array}\right)
$$

for $1 \leq i \leq n-1$, and the following $n^{2}$ relations:

(A) $v_{n-i} u_{i}=u_{i} v_{n-i+1}$, for $2 \leq i \leq n-1$;

$\left(A_{2}\right) u_{n-i} v_{i}=v_{i} u_{n-i+1}$, for $2 \leq i \leq n-1$;

(A) $v_{n-i} u_{i}=u_{i}$, for $1 \leq i \leq n-1$;

(A $\left.A_{4}\right) u_{n-i} v_{i}=v_{i}$, for $1 \leq i \leq n-1$;

$\left(A_{5}\right) u_{i} v_{j}=v_{j} u_{i}$, for $1 \leq i, j \leq n-1$, with $j \notin\{n-i, n-i+1\}$;

(A6) $u_{1} u_{2} u_{1}=u_{1} u_{2}$

$\left(A_{7}\right) v_{1} v_{2} v_{1}=v_{1} v_{2}$.

Recall that $\mathscr{G D _ { n }}$ is the monoid of all full transformations of $X_{n}$ that preserve or reverse the order, of which $\mathscr{G}_{n}$ is a submonoid. Consider the following permutation of order two:

$$
h=\left(\begin{array}{ccccc}
1 & 2 & \cdots & n-1 & n \\
n & n-1 & \cdots & 2 & 1
\end{array}\right) .
$$

Clearly, $h$ is an element of $\mathscr{O} \mathscr{D}_{n}$. On the other hand, given an order reversing full transformation $s$, we have $s h \in \mathscr{O}_{n}$, whence $s h=s_{1} s_{2} \cdots s_{k}$, for some $s_{1}, s_{2}, \ldots, s_{k} \in X$ and $k \in \mathbb{N}$. Thus $s=s h^{2}=s_{1} s_{2} \cdots s_{k} h$, and we can conclude the following.

Proposition 2.1. The set $Y=X \cup\{h\}$ generates $\mathscr{O Q}_{n}$.

Now consider the following set of monoid relations:

$\left(N A_{0}\right) h^{2}=1$;

$\left(N A_{1}\right) h u_{i}=v_{i} h$, for all $i \in\{1, \ldots, n-1\}$;

$\left(N A_{2}\right) v_{1} v_{2} \cdots v_{n-1} h=v_{1} v_{2} \cdots v_{n-1} u_{1} u_{2} \cdots u_{n-1}$. 
It is a routine matter to prove that these relations over the alphabet $Y$ are satisfied by the generating set $Y$ of $\mathscr{O} \mathscr{D}_{n}$.

Next, define $\bar{A}=A \cup N A_{0} \cup N A_{1} \cup N A_{2}$. Observe that $|\bar{A}|=n^{2}+n+1$.

As $\langle X \mid A\rangle$ is a presentation for $\mathscr{O}_{n}$, we can take a set $W^{\prime}$ of forms for $\mathscr{G}_{n}$ (associated to this presentation).

Let

$$
\alpha_{i}=\left(\begin{array}{ccc}
1 & \cdots & n \\
i & \cdots & i
\end{array}\right) \in \mathscr{O}_{n}, \quad \text { for } 1 \leq i \leq n
$$

Then it is easy to show that $\alpha_{i}=v_{1} v_{2} \cdots v_{n-1} u_{1} u_{2} \cdots u_{i-1}$ (for $i=1$ the expression $u_{1} u_{2} \cdots u_{i-1}$ denotes the identity).

Let $\beta_{i}=u_{1} \cdots u_{i-1}$, for $1 \leq i \leq n$. Let $u_{0}, w_{1}, \ldots, w_{n} \in W^{\prime}$ be the words that represent the elements $\alpha_{1}, \beta_{1}, \ldots, \beta_{n} \in \mathscr{O}_{n}$, respectively.

Consider $W_{\alpha}=\left\{u_{0} w_{i}: 1 \leq i \leq n\right\} \quad$ and $\quad W_{\beta}=\left\{w \in W^{\prime}: w \varphi \in J_{2} \cup \cdots \cup J_{n}\right\}$, where $\varphi$ denotes the canonical morphism from $X^{*}$ onto $\mathscr{O}_{n}$, and each $J_{k}$, for $2 \leq k \leq n$, denotes the $\mathcal{F}$-class of all elements of $\mathscr{O}_{n}$ with rank $k$. Then $W=W_{\alpha} \cup W_{\beta}$ is a new set of forms for $\mathscr{G}_{n}$, where $W_{\alpha}$ is the set of forms that represents the constant transformations of $\mathscr{O}_{n}$.

Now let us take $\bar{W}=W \cup\left\{w h: w \in W_{\beta}\right\}$. Notice that

$$
|\bar{W}|=2\left|\mathscr{O}_{n}\right|-n=\left|\mathscr{O}_{n}\right|,
$$

and that $u_{0}$ is a (left) factor of each word in $W_{\alpha}$. Since $W$ must contain the empty word, by Theorem 1.3, we conclude that the following result holds.

Theorem 2.2. The monoid $\mathscr{O Q D}_{n}$ is defined by the presentation $\langle Y \mid \bar{A}\rangle$, on $2 n-1$ generators and $n^{2}+n+1$ relations.

Next we aim to improve this presentation for $\mathscr{O}_{n}$. By applying Tietze transformations to $\langle Y \mid \bar{A}\rangle$, we will find a new presentation with just $n$ generators and $\left(n^{2}+n+2\right) / 2$ relations.

First observe that the relations $h u_{i}=v_{i} h$ and $h u_{i} h=v_{i}$, for $1 \leq i \leq n-1$, are equivalent, by $\left(N A_{0}\right)$. Hence, making the necessary substitutions, the letters $v_{1}, \ldots, v_{n-1}$ can be eliminated together with the relations $h u_{i}=v_{i} h$, for $1 \leq i \leq n-1$. On the other hand, we can replace relations $\left(A_{1}\right)$ and $\left(A_{2}\right)$ by relations

$\left(A_{1}^{\prime}\right) \quad h u_{n-i} h u_{i}=u_{i} h u_{n-i+1} h$, for $2 \leq i \leq n-1$;

relations $\left(A_{3}\right)$ and $\left(A_{4}\right)$, by relations

$\left(A_{2}^{\prime}\right) h u_{n-i} h u_{i}=u_{i}$, for $1 \leq i \leq n-1$;

and relations $\left(A_{6}\right)$ and $\left(A_{7}\right)$, by the relation

$\left(A_{4}^{\prime}\right) u_{1} u_{2} u_{1}=u_{1} u_{2}$.

Relations $u_{i} v_{j}=v_{j} u_{i}$ and $u_{i} h u_{j} h=h u_{j} h u_{i}$, for $1 \leq i, j \leq n-1$ with $j \notin\{n-i$, $n-i+1\}$, are equivalent in view of $\left(N A_{1}\right)$ and $\left(N A_{0}\right)$; by $\left(N A_{0}\right)$, so are the relations $u_{i} h u_{j} h=h u_{j} h u_{i}$ and $u_{j} h u_{i} h=h u_{i} h u_{j}$, for $1 \leq i, j \leq n-1$ with $j \notin\{n-i, n-i+1\}$. 
Hence we can replace relations $\left(A_{5}\right)$ by relations

$\left(A_{3}^{\prime}\right) u_{i} h u_{j} h=h u_{j} h u_{i}$, for $j \in\{i, \ldots, n-i-1\} \quad$ if $1 \leq i \leq\left\lceil\frac{n-1}{2}\right\rceil-1$, and for $j \in$ $\{n-i+2, \ldots, i\}$ if $\left\lceil\frac{n-1}{2}\right\rceil+2 \leq i \leq n-1$;

$u_{\left\lceil\frac{n}{2}\right\rceil+(-1)^{n}} h u_{\left\lceil\frac{n}{2}\right\rceil+(-1)^{n}} h=h u_{\left\lceil\frac{n}{2}\right\rceil+(-1)^{n}} h u_{\left\lceil\frac{n}{2}\right\rceil+(-1)^{n}}$.

Recall that, for $x \in \mathbb{R}$, expression $\lceil x\rceil$ denotes the least integer greater than or equal to $x$.

Making the necessary substitutions, we can replace the relation $\left(N A_{2}^{\prime}\right)$ by the relation

$\left(N A_{2}^{\prime}\right) h u_{1} \cdots u_{n-1}=\left(h u_{1} \cdots u_{n-1}\right)^{2}$.

Therefore, using Tietze transformations, we concluded that $\left\langle Y^{\prime} \mid \bar{A}^{\prime}\right\rangle$, where $Y^{\prime}=\left\{u_{1}, \ldots, u_{n-1}, h\right\}$ and $\bar{A}^{\prime}=A_{1}^{\prime} \cup A_{2}^{\prime} \cup A_{3}^{\prime} \cup A_{4}^{\prime} \cup N A_{0} \cup N A_{2}^{\prime}$, is a new presentation for $\mathscr{O}_{\mathscr{D}_{n}}$. Since $\left|\bar{A}^{\prime}\right|=(1 / 2)\left(n^{2}+n+2\right)$, we have the following corollary:

Corollary 2.3. The monoid $\mathscr{O Q D}_{n}$ admits a presentation with $n$ generators and $\left(n^{2}+n+2\right) / 2$ relations.

\section{A PRESENTATION FOR $\mathscr{P} \mathscr{O} \mathscr{D}_{n}$}

In this section we aim to obtain presentations for the monoid $\mathscr{P} \mathscr{O} \mathscr{D}_{n}$ of all partial transformations on $X_{n}$ that preserve or reverse the order. We treat the partial case similarly to the way we dealt with the full case.

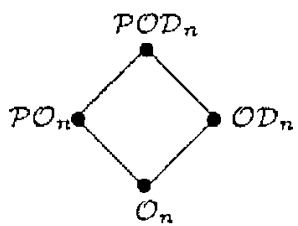

So, as for $\mathscr{G Q D}_{n}$, we use the method given by Theorem 1.3, applying it to a presentation of the submonoid $\mathscr{P}_{n}$ of all partial transformations on $X_{n}$ that preserve order. Fernandes (2002a) observed that the pair $\langle Y \mid P\rangle$ described below is a presentation for $\mathscr{P O}_{n}$. Take $Y=\left\{u_{1}, \ldots, u_{n-1}, v_{1}, \ldots, v_{n-1}, c_{1}, \ldots, c_{n}\right\}$, with $u_{i}$ and $v_{i}$, for $1 \leq i \leq n-1$, defined as in Section 2, and

$$
c_{i}=\left(\begin{array}{ccc|ccc}
1 & \cdots & i-1 & i+1 & \cdots & n \\
1 & \cdots & i-1 & i+1 & \cdots & n
\end{array}\right),
$$

for $1 \leq i \leq n$. Then $Y$ is a generating set of the monoid $\mathscr{P} \mathscr{O}_{n}$ consisting of $3 n-2$ idempotents. Let $P$ be the set consisting of the following $\left(7 n^{2}-n-4\right) / 2$ relations over the alphabet $Y$ :

(A $) v_{n-i} u_{i}=u_{i} v_{n-i+1}$, for $2 \leq i \leq n-1$;

$\left(A_{2}\right) u_{n-i} v_{i}=v_{i} u_{n-i+1}$, for $2 \leq i \leq n-1$; 
(A) $v_{n-i} u_{i}=u_{i}$, for $1 \leq i \leq n-1$;

(A $\left.A_{4}\right) u_{n-i} v_{i}=v_{i}$, for $1 \leq i \leq n-1$;

(A) $u_{i} v_{j}=v_{j} u_{i}$, for $1 \leq i, j \leq n-1$, with $j \notin\{n-i, n-i+1\}$;

(A) $u_{1} u_{2} u_{1}=u_{1} u_{2}$

$\left(A_{7}\right) v_{1} v_{2} v_{1}=v_{1} v_{2}$;

(E $\left.E_{1}\right) c_{i} c_{j}=c_{j} c_{i}$, for $1 \leq i<j \leq n$;

$\left(P_{1}\right) u_{i} c_{i}=u_{i}$, for $1 \leq i \leq n-1$;

$\left(P_{2}\right) c_{i} u_{i}=c_{i}$, for $1 \leq i \leq n-1$;

$\left(P_{3}\right) u_{i} c_{i+1}=c_{i} c_{i+1}$, for $1 \leq i \leq n-1$;

$\left(P_{4}\right) c_{i} u_{j}=u_{j} c_{i}$, for $1 \leq i, j \leq n-1$ such that $i \notin\{j, j+1\}$;

$\left(P_{4}^{\prime}\right) c_{n} u_{j}=u_{j} c_{n}$, for $1 \leq j \leq n-2$;

(P) $v_{n-i} c_{i+1}=v_{n-i}$, for $1 \leq i \leq n-1$;

( $\left.P_{6}\right) c_{i+1} v_{n-i}=c_{i+1}$, for $1 \leq i \leq n-1$;

$\left(P_{7}\right) v_{n-i} c_{i}=c_{i+1} c_{i}$, for $1 \leq i \leq n-1$;

$\left(P_{8}\right) c_{i} v_{n-j}=v_{n-j} c_{i}$, for $1 \leq i, j \leq n-1$ such that $i \notin\{j, j+1\}$;

$\left(P_{8}^{\prime}\right) c_{n} v_{n-j}=v_{n-j} c_{n}$, for $1 \leq j \leq n-2$.

An argument similar to the one used to prove Proposition 2.1 allows us to state the following.

Proposition 3.1. The set $Y^{\prime}=Y \cup\{h\}$ generates $\mathscr{P} \mathscr{O} \mathscr{D}_{n}$.

Let us consider the following set of monoid relations over the alphabet $Y^{\prime}$ :

$\left(N P_{0}\right) h^{2}=1$;

$\left(N P_{1}\right) h u_{i}=v_{i} h$, for $1 \leq i \leq n-1$;

$\left(N P_{2}\right) h c_{i}=c_{n-i+1} h$, for $1 \leq i \leq n$;

$\left(N P_{3}\right) v_{1} v_{2} \cdots v_{n-1} h=v_{1} v_{2} \cdots v_{n-1} u_{1} u_{2} \cdots u_{n-1}$.

These relations are satisfied by the generating set $Y^{\prime}$ of $\mathscr{P} \mathscr{O} \mathscr{D}_{n}$. Next, define $\bar{P}=P \cup$ $N P_{0} \cup N P_{1} \cup N P_{2} \cup N P_{3}$ and observe that $|\bar{P}|=\left(7 n^{2}+3 n-2\right) / 2$.

Let $W^{\prime}$ be a set of forms for $\mathscr{P} \mathscr{C}_{n}$, with regard to its presentation $\langle Y \mid P\rangle$. Notice that the empty word belongs to $W^{\prime}$.

Let $\alpha_{i}^{i_{1}<\cdots<i_{k}}$ be the constant transformation of $\mathscr{P} \mathscr{O}_{n}$ with domain $\{1, \ldots, n\} \backslash\left\{i_{1}, \ldots, i_{k}\right\}$ and image $\{i\}$, for $i \in\{1, \ldots, n\}$ and $k \in\{1, \ldots, n-1\}$. For $k=0$, define $\alpha_{i}^{i_{1}<\cdots<i_{k}}$ as being the full constant transformation with image $\{i\}$. It is easy to prove that

$$
\alpha_{i}^{i_{1}<\cdots<i_{k}}=c_{i_{1}} \cdots c_{i_{k}} v_{1} \cdots v_{n-1} u_{1} \cdots u_{i-1},
$$

where $c_{i_{1}} \cdots c_{i_{k}}$ denotes the identity, if $k=0$. Denote by $\beta_{i}$ the product $u_{1} \cdots u_{i-1}$, for $2 \leq i \leq n$, and by $\beta_{1}$ the identity. Let $u_{0}, w_{1}, \ldots, w_{n}, w_{i_{1}<\cdots<i_{k}} \in W^{\prime}$ be the words that represent the elements $v_{1} \cdots v_{n-1}, \beta_{1}, \ldots, \beta_{n}, c_{i_{1}} \cdots c_{i_{k}} \in \mathscr{P} \mathscr{O}_{n}$, respectively. For $k=0$ let $w_{i_{1}<\cdots<i_{k}}$ be the empty word (i.e., $w_{1}$ ). Notice that if $v_{0} \in W^{\prime}$ is the word that represents the empty transformation, then the word $u_{0} v_{0}$ also represents the empty transformation.

Consider $W_{\alpha}=\left\{w_{i_{1}<\cdots<i_{k}} u_{0} w_{i}: 1 \leq i \leq n\right.$ and $\left.0 \leq k \leq n-1\right\} \cup\left\{u_{0} v_{0}\right\}$ and $W_{\beta}=$ $\left\{w \in W^{\prime}: w \varphi \in J_{2} \cup \cdots \cup J_{n}\right\}$, where $\varphi$ is the canonical morphism from $Y^{*}$ onto $\mathscr{P} \mathscr{O}_{n}$. Then $W=W_{\alpha} \cup W_{\beta}$ is a new set of forms of $\mathscr{P} \mathscr{G}_{n}$. 
Finally, take $\bar{W}=W \cup\left\{w h: w \in W_{\beta}\right\}$. Notice that

$$
|\bar{W}|=2\left|\mathscr{P} \mathscr{O}_{n}\right|-\left(1+\sum_{r=1}^{n}\left(\begin{array}{l}
n \\
r
\end{array}\right) n\right)=\left|\mathscr{P} \mathscr{O} \mathscr{D}_{n}\right|,
$$

and $u_{0}$ is a factor of each word in $W_{\alpha}$. Hence, by Theorem 1.3, we have the following result.

Theorem 3.2. The monoid $\mathscr{P} \mathscr{C} \mathscr{D}_{n}$ is defined by the presentation $\left\langle Y^{\prime} \mid \bar{P}\right\rangle$, on $3 n-2$ generators and $\left(7 n^{2}+3 n-2\right) / 2$ relations.

Next we show how to improve this presentation of $\mathscr{P} \mathscr{O} \mathscr{D}_{n}$.

The relations $h u_{i}=v_{i} h$ and $h u_{i} h=v_{i}$, for $1 \leq i \leq n-1$, are equivalent. Hence, making the necessary substitutions, the letters $v_{1}, \ldots, v_{n-1}$ can be eliminated together with the relations $h u_{i}=v_{i} h$, for $1 \leq i \leq n-1$. On the other hand, the relations $h c_{i}=c_{n-i+1} h$ and $c_{n-i+1}=h c_{i} h$, for $1 \leq i \leq n$, are also equivalent. Thus, making the necessary substitutions, the letters $c_{[n / 2\rceil+1}, \ldots, c_{n}$ can be eliminated. If $n$ is even it is also possible to eliminate all $\left(N P_{2}\right)$ relations. If $n$ is odd we eliminate all $\left(N P_{2}\right)$ relations except the relation $h c_{\lceil n / 2\rceil}=c_{\lceil n / 2\rceil} h$ that we denote by $\left(N P_{2}^{\prime}\right)$.

On the other hand, it is easy to show that relations $\left(P_{5}\right)$ to $\left(P_{8}\right)$ and $\left(P_{8}^{\prime}\right)$ follow from relations $\left(P_{1}\right)$ to $\left(P_{4}\right),\left(P_{4}^{\prime}\right)$ and $\left(N P_{0}\right),\left(N P_{1}\right),\left(N P_{2}\right)$.

Now, as consequence of the calculations done for the monoid $\mathscr{C O D}_{n}$ and of the considerations above, we conclude that the monoid $\mathscr{P} \mathscr{C} \mathscr{D}_{n}$ admits a presentation $\left\langle Z \mid P^{\prime}\right\rangle$ in terms of the $\lceil n / 2\rceil+n$ generating set

$$
Z=\left\{u_{1}, \ldots, u_{n-1}, c_{1}, \ldots, c_{\lceil n / 2\rceil}, h\right\}
$$

and the set of relations $P^{\prime}$ defined by

$\left(A_{1}^{\prime}\right) h u_{n-i} h u_{i}=u_{i} h u_{n-i+1} h$, for $2 \leq i \leq n-1$;

$\left(A_{2}^{\prime}\right) h u_{n-i} h u_{i}=u_{i}$, for $1 \leq i \leq n-1$;

$\left(A_{3}^{\prime}\right) u_{i} h u_{j} h=h u_{j} h u_{i}$, for $j \in\{i, \ldots, n-i-1\}$ if $1 \leq i \leq\lceil(n-1) / 2\rceil-1$, and

for $j \in\{n-i+2, \ldots, i\}$ if $\lceil(n-1) / 2\rceil+2 \leq i \leq n-1$;

$\left(A_{4}^{\prime}\right) u_{1} u_{2} u_{1}=u_{1} u_{2}$

$u_{\lceil n / 2\rceil+(-1)^{n}} h u_{\lceil n / 2\rceil+(-1)^{n}} h=h u_{\lceil n / 2\rceil+(-1)^{n}} h u_{\lceil n / 2\rceil+(-1)^{n}}$;

$\left(E_{1}^{\prime}\right) c_{i} c_{j}=c_{j} c_{i}$, for $1 \leq i \leq\lceil n / 2\rceil-1$ and $i+1 \leq j \leq\lceil n / 2\rceil$;

$c_{i} h c_{j} h=h c_{j} h c_{i}$, for $1 \leq i \leq\lceil n / 2\rceil$ and $i \leq j \leq\lceil n / 2\rceil$

$(1 \leq i \leq\lceil n / 2\rceil-1$ and $i \leq j \leq\lceil n / 2\rceil-1$ if $n$ is odd $)$;

$\left(P_{1}^{\prime}\right) u_{i} c_{i}=u_{i}$, for $1 \leq i \leq\lceil n / 2\rceil$

$u_{i} h c_{n-i+1}=u_{i} h$, for $\lceil n / 2\rceil+1 \leq i \leq n-1$;

$\left(P_{2}^{\prime}\right) c_{i} u_{i}=c_{i}$, for $1 \leq i \leq\lceil n / 2\rceil$

$c_{n-i+1} h u_{i}=c_{n-i+1} h$, for $\lceil n / 2\rceil+1 \leq i \leq n-1$;

$\left(P_{3}^{\prime}\right) u_{i} c_{i+1}=c_{i} c_{i+1}$, for $1 \leq i \leq\lceil n / 2\rceil-1$

$u_{i} h c_{n-i}=h c_{n-i+1} c_{n-i}$, for $\lceil n / 2\rceil \leq i \leq n-1$;

$\left(P_{4}^{*}\right) c_{i} u_{j}=u_{j} c_{i}$, for $1 \leq i, j \leq n-1$ such that $i \notin\{j, j+1\}$ and $i \leq\lceil n / 2\rceil$

$h c_{n-i+1} h u_{j}=u_{j} h c_{n-i+1} h$, for $i>\lceil n / 2\rceil ;$

$\left(P_{4}^{\prime \prime}\right) h c_{1} h u_{j}=u_{j} h c_{1} h$, for $1 \leq j \leq n-2$;

$\left(N P_{0}\right) h^{2}=1$; 
$\left(N P_{2}^{\prime}\right) h c_{\lceil n / 2\rceil}=c_{\lceil n / 2\rceil} h$, if $n$ is odd;

$\left(N P_{3}^{\prime}\right) h u_{1} \cdots u_{n-1}=\left(h u_{1} \cdots u_{n-1}\right)^{2}$.

It is a routine matter to show that $P^{\prime}$ has $\left(7 n^{2}+2 n\right) / 4$ elements if $n$ is even, and $\left(7 n^{2}+2 n+3\right) / 4$ elements if $n$ is odd.

Corollary 3.3. The monoid $\mathscr{P O P O}_{n}$ admits a presentation with $\lceil n / 2\rceil+n$ generators and $\left\{7 n^{2}+2 n+(3 / 2)\left[1-(-1)^{n}\right]\right\} / 4$ relations.

\section{A PRESENTATION FOR $\mathscr{P} \mathscr{O} \mathscr{P}_{n}$}

Another natural semigroup to consider is $\mathscr{\mathscr { P } \mathscr { P }} \mathscr{P}_{n}$, the monoid of all orientationpreserving partial transformations on $X_{n}$. To obtain a presentation for $\mathscr{P} \mathscr{O} \mathscr{P}_{n}$ is considerably more complicated than in the previous cases. The technique used, however, is based on Theorem 1.1, but in this case we will need to make use simultaneously of presentations for the submonoids $\mathscr{P O} \mathscr{C}_{n}$ and $\mathscr{G} \mathscr{P}_{n}$ of $\mathscr{P} \mathscr{O} \mathscr{P}{ }_{n}$.

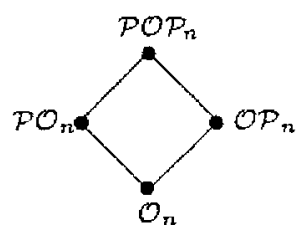

Let $\langle X \mid R\rangle$ be any presentation for the monoid $\mathscr{O} \mathscr{P}_{n}$, in terms of the $(2 n-1)$ element generating set

$$
X=\left\{u_{1}, \ldots, u_{n-1}, v_{1}, \ldots, v_{n-1}, g\right\},
$$

where $u_{1}, \ldots, u_{n-1}, v_{1}, \ldots, v_{n-1}$ are defined in Section 2 and $g$ is the $n$-cycle

$$
\left(\begin{array}{ccccc}
1 & 2 & \cdots & n-1 & n \\
2 & 3 & \cdots & n & 1
\end{array}\right) .
$$

Let $\langle Y \mid P\rangle$ be the presentation for the monoid $\mathscr{P} \mathscr{C}_{n}$ already considered in Section 3.

Let $C=\left\{c_{1}, \ldots, c_{n}\right\}$ and consider the following set $N$ of relations over the alphabet $C \cup\{g\}$ :

$$
N=\left\{g c_{i}=c_{i-1} g, 2 \leq i \leq n ; g c_{1}=c_{n} g\right\} .
$$

Let $Z=X \cup Y=X \cup\left\{c_{1}, \ldots, c_{n}\right\}=Y \cup\{g\}$.

Let $\mathscr{E}_{n}=\langle C\rangle$ be the semilattice generated by $C$.

We begin by proving the following.

Proposition 4.1. For all $w \in Z^{*}$, there exist $c \in C^{*}$ and $u \in X^{*}$ such that the relation $w=c u$ is a consequence of $P$ and $N$.

To prove this result we first present a couple of lemmas. 
As usual, given a word $w$, we denote its length by $|w|$. It is a routine matter to prove:

Lemma 4.2. Let $x \in X$ and $c \in C$. Then there exist $c^{\prime} \in C^{*}$ and $x^{\prime} \in X^{*}$, with $1 \leq\left|c^{\prime} x^{\prime}\right| \leq 2$ and $\left|x^{\prime}\right| \leq 1$, such that the relation $x c=c^{\prime} x^{\prime}$ is a consequence of $P$ and $N$.

Lemma 4.3. Let $x \in X$ and $c \in C^{*}$. There exist $c^{\prime} \in C^{*}$ and $x^{\prime} \in X^{*}$ such that the relation $x c=c^{\prime} x^{\prime}$ is a consequence of relations $P$ and $N$.

Proof. We prove this result by induction on $|c|$. If $|c|=1$ then, by Lemma 4.2, there exist $c^{\prime} \in C^{*}$ and $x^{\prime} \in X^{*}$, with $1 \leq\left|c^{\prime} x^{\prime}\right| \leq 2$ and $\left|x^{\prime}\right| \leq 1$, such that the relation $x c=c^{\prime} x^{\prime}$ is a consequence of $P$ and $N$. Now let $|c|=n+1$, with $n \in \mathbb{N}$. Then $c=c_{i} c^{\prime}$, for some $1 \leq i \leq n$ and $c^{\prime} \in C^{*}$ such that $\left|c^{\prime}\right|=n$. By Lemma 4.2 and the induction hypothesis, we have

$$
x c=x\left(c_{i} c^{\prime}\right)=\left(x c_{i}\right) c^{\prime}=\left(c^{\prime \prime} x^{\prime}\right) c^{\prime}=c^{\prime \prime}\left(x^{\prime} c^{\prime}\right)=c^{\prime \prime}\left(c^{\prime \prime \prime} x^{\prime \prime}\right)=\left(c^{\prime \prime} c^{\prime \prime \prime}\right) x^{\prime \prime},
$$

for some $x^{\prime} \in X^{*}, x^{\prime \prime} \in X^{*}$ and $c^{\prime \prime}, c^{\prime \prime \prime} \in C^{*}$. Thus the result follows.

Now, we can prove Proposition 4.1.

Proof of Proposition 4.1. We will proceed by induction on $|w|$. Let $w \in Z^{*}$. If $|w| \in\{0,1\}$, the result is trivially true. Suppose that $|w|=n+1$, with $n \in \mathbb{N}$. Take $w^{\prime} \in Z^{*}$, with $\left|w^{\prime}\right|=n$ and $y \in Z$ such that $w=y w^{\prime}$. By the induction hypothesis, we have $w^{\prime}=c u$, for some $u \in X^{*}$ and $c \in C^{*}$. Thus $w=y(c u)$. Now, by Lemma 4.3, there exist $y^{\prime} \in X^{*}$ and $c^{\prime} \in C^{*}$ such that $y c=c^{\prime} y^{\prime}$. Hence $w=(y c) u=\left(c^{\prime} y^{\prime}\right) u=$ $c^{\prime}\left(y^{\prime} u\right)$ and the result follows.

Next we aim to prove that $\langle Z \mid R, P, N\rangle$ is a presentation for $\mathscr{P} \mathscr{O} \mathscr{P}_{n}$. We start by making a series of remarks.

Let $1 \leq k \leq n$ and $i_{1}, \ldots, i_{k} \in\{1, \ldots, n\}$ be such that $i_{1}<\cdots<i_{k}$. Consider the following idempotent of $\mathscr{O}_{n}$ :

$$
\delta_{i_{1}, \ldots, i_{k}}=\left(\begin{array}{ccc|c|ccc|ccc}
1 & \cdots & i_{1} & \cdots & i_{k-1}+1 & \cdots & i_{k} & i_{k}+1 & \cdots & n \\
i_{1} & \cdots & i_{1} & \cdots & i_{k} & \cdots & i_{k} & i_{k} & \cdots & i_{k}
\end{array}\right) .
$$

Let

$$
c_{i_{1}, \ldots, i_{k}}=\prod_{\substack{i=1 \\
i \neq i_{1}, \ldots, i_{k}}}^{n} c_{i}=\left(\begin{array}{lll}
i_{1} & \cdots & i_{k} \\
i_{1} & \cdots & i_{k}
\end{array}\right) \in E\left(\mathscr{I}_{n}\right)
$$

Clearly $c_{i_{1}, \ldots, i_{k}}=c_{i_{1}, \ldots, i_{k}} \delta_{i_{1}, \ldots, i_{k}} \in \mathscr{P} \mathscr{O}_{n}$. Notice that if $k=n$, then $c_{i_{1}, \ldots, i_{k}}=1$.

For each cyclic sequence $\left(j_{1}, \ldots, j_{k}\right)$, let

$$
\Delta_{j_{1}, \ldots, j_{k}}^{i_{1}, \ldots, i_{k}}=\left\{\alpha \in \mathscr{G} \mathscr{P}_{n} \mid i_{t} \alpha=j_{t}, 1 \leq t \leq k\right\}
$$


Remark 4.4. For all $\alpha, \beta \in \Delta_{j_{1}, \ldots, j_{k}}^{i_{1}, \ldots, i_{k}}$, we have $\delta_{i_{1}, \ldots, i_{k}} \alpha=\delta_{i_{1} \ldots, i_{k}} \beta \in \Delta_{j_{1}, \ldots, j_{k}}^{i_{1}, \ldots, i_{k}}$. We denote this element of $\Delta_{j_{1}, \ldots, j_{k}}^{i_{1}, \ldots, i_{k}}$ by $\delta_{j_{1}, \ldots, j_{k}}^{i_{1}, \ldots, i_{k}}$. Observe that

$$
\delta_{j_{1}, \ldots, j_{k}}^{i_{1}, \ldots, i_{k}}=\left(\begin{array}{ccc|c|ccc|ccc}
1 & \cdots & i_{1} & \cdots & i_{k-1}+1 & \cdots & i_{k} & i_{k}+1 & \cdots & n \\
j_{1} & \cdots & j_{1} & \cdots & j_{k} & \cdots & j_{k} & j_{k} & \cdots & j_{k}
\end{array}\right) .
$$

Remark 4.5. By definition of $\delta_{j_{1}, \ldots, j_{k}}^{i_{1}, \ldots, i_{k}}$, we have $\delta_{i_{1}, \ldots, i_{k}} \delta_{j_{1}, \ldots, j_{k}}^{i_{1}, \ldots, i_{k}}=\delta_{j_{1}, \ldots, j_{k}}^{i_{1}, \ldots, i_{k}}$ in $\mathscr{G} \mathscr{P}_{n}$.

Let $\alpha \in \mathscr{P} \mathscr{O} \mathscr{P}_{n}$, with $\operatorname{Dom}(\alpha)=\left\{i_{1}<\cdots<i_{k}\right\}$ and cyclic sequence of images $\left(j_{1}, \ldots, j_{k}\right)=\left(i_{1} \alpha, \ldots, i_{k} \alpha\right)$. Then $\alpha=c_{i_{1}, \ldots, i_{k}} \delta_{j_{1}, \ldots, j_{k}}^{i_{1}, \ldots, i_{k}}$ and so $\mathscr{P} \mathscr{O P}{ }_{n}$ is generated by $Z$.

We have proved that any nonzero element $\alpha \in \mathscr{P} \mathscr{C} \mathscr{P}{ }_{n}$ can be written in the form $c_{i_{1}, \ldots, i_{k}} \delta_{j_{1}, \ldots, i_{k}}^{i_{1}, \ldots, i_{k}}$. Next we show that such expression is unique.

We begin by proving the following lemma.

Lemma 4.6. For all $\gamma_{1}, \gamma_{2} \in \mathscr{E}_{n}$ and $\beta_{1}, \beta_{2} \in \mathscr{O P}_{n}$, if $\gamma_{1} \beta_{1}=\gamma_{2} \beta_{2}$, then $\gamma_{1}=\gamma_{2}$.

Proof. As $\operatorname{Dom}\left(\gamma_{i} \beta_{i}\right)=\operatorname{Dom}\left(\gamma_{i}\right)$, for $i \in\{1,2\}$, then $\operatorname{Dom}\left(\gamma_{1}\right)=\operatorname{Dom}\left(\gamma_{2}\right)$. Hence $\gamma_{1}=\gamma_{2}$, since $\gamma_{1}$ and $\gamma_{2}$ are partial identities.

Now suppose that we also have $\alpha=c_{i_{1}^{\prime}, \ldots, i_{k^{\prime}}} \beta$, with $\beta=\delta_{j_{1}^{\prime}, \ldots, j_{k^{\prime}}^{\prime}}^{i_{1}^{\prime}, \ldots, i_{k^{\prime}}^{\prime}} \in \mathscr{O} \mathscr{P}_{n}$, for some $1 \leq i_{1}^{\prime}<\cdots<i_{k^{\prime}}^{\prime} \leq n$ and a cyclic sequence $\left(j_{1}^{\prime}, \ldots, j_{k^{\prime}}^{\prime}\right)$. Then, by Lemma 4.6, we must have $c_{i_{1}, \ldots, i_{k}}=c_{i_{1}^{\prime}, \ldots, i_{k^{\prime}}^{\prime}}$, which are partial identities. Hence $k=k^{\prime}$ and $i_{t}^{\prime}=i_{t}$, for $1 \leq t \leq k$. Therefore

$$
\alpha=c_{i_{1}, \ldots, i_{k}} \delta_{j_{1}, \ldots, j_{k}}^{i_{1}, \ldots, i_{k}}=c_{i_{1}, \ldots, i_{k}} \delta_{j_{1}^{\prime}, \ldots, j_{k}^{\prime}}^{i_{1}, \ldots, i_{k}}
$$

whence $j_{t}^{\prime}=i_{t} \delta_{j_{1}^{\prime}, \ldots, j_{k}^{\prime}}^{i_{1}, \ldots, i_{k}}=i_{t} \delta_{j_{1}, \ldots, j_{k}}^{i_{1}, \ldots, i_{k}}=j_{t}$, for $1 \leq t \leq k$, and so we also have $\beta=\delta_{j_{1}, \ldots, j_{k}}^{i_{1}, \ldots, i_{k}}$.

Thus each nonzero element $\alpha \in \mathscr{P} \mathscr{O P}{ }_{n}$ can be written uniquely in the form $\alpha=$ $c_{i_{1}, \ldots, i_{k}} \delta_{j_{1}, \ldots, j_{k}}^{i_{1}, \ldots, i_{k}}$.

Let $\mathrm{W}$ be a set of forms for $\mathscr{C O P}_{n}$ corresponding to the presentation $\langle X \mid R\rangle$. For each $1 \leq i_{1}<\cdots<i_{k} \leq n$ and each cyclic sequence $\left(j_{1}, \ldots, j_{k}\right)$, with $1 \leq k \leq n$, let $w_{j_{1}, \ldots, j_{k}}^{i_{1}, \ldots, i_{k}} \in W$ be the word that represents $\delta_{j_{1}, \ldots, j_{k}}^{i_{1}, \ldots, i_{k}}$.

Let $\bar{W} \subseteq Z^{*}$ be the set of words of the form

$$
\left(\prod_{\substack{i=1 \\ i \neq i_{1}, \ldots, i_{k}}}^{n} c_{i}\right) w_{j_{1}, \ldots, j_{k}}^{i_{1}, \ldots, i_{k}},
$$

with $1 \leq i_{1}<\cdots<i_{k} \leq n,\left(j_{1}, \ldots, j_{k}\right)$ a cyclic sequence and $1 \leq k \leq n$, together with the word $c_{1} \cdots c_{n}$ (representing the zero).

Clearly, we have $|\bar{W}|=\left|\mathscr{P} \mathscr{P}_{n}\right|$.

Now, we are in a position to obtain a presentation for $\mathscr{P} \mathscr{G} \mathscr{P}_{n}$.

Proposition 4.7. The monoid $\mathscr{P} \mathscr{C P}_{n}$ is defined by the presentation $\langle Z \mid R, P, N\rangle$.

Proof. In view of the previous results, it remains to prove condition (2) of Theorem 1.1. Let $w \in Z^{*}$. Then, by Proposition 4.1, there exist $c \in C^{*}$ and $u \in X^{*}$ 
such that the relation $w=c u$ is a consequence of $P$ and $N$. Also, for some $1 \leq i_{1}<$ $\cdots<i_{k} \leq n$ and $0 \leq k \leq n$, we can obtain the relation

$$
c=\prod_{\substack{i=1 \\ i \neq i_{1}, \ldots, i_{k}}}^{n} c_{i}
$$

as a consequence of $P$.

First, we suppose that $k=0$, i.e., we have the relation $c=c_{1} \cdots c_{n}$.

Let us prove that the relation $c_{1} \cdots c_{n} u=c_{1} \cdots c_{n}$ is a consequence of $P$ and $R$. We may consider $|u| \geq 1$ and proceed by induction on $|u|$.

Suppose that $|u|=1$. Since we have the equality $v_{1} g=u_{n-1} \cdots u_{1}$ in $\mathscr{O O P}_{n}$ and $\langle X \mid R\rangle$ is a presentation for $\mathscr{G P}_{n}$, the relation $v_{1} g=u_{n-1} \cdots u_{1}$ is a consequence of $R$. On the other hand, we have the relation $c_{n}=c_{n} v_{1}$ in $P$, whence

$$
c_{1} \cdots c_{n} g=c_{1} \cdots c_{n} v_{1} g=c_{1} \cdots c_{n} u_{n-1} \cdots u_{1} \text {. }
$$

Now, as $c_{1} \cdots c_{n}$ represents the zero of $\mathscr{P} \mathscr{O}_{n}$ and $u_{n-1} \cdots u_{1}$ represents an element of $\mathscr{P} \mathscr{C}_{n}$, the relation $c_{1} \cdots c_{n} u_{n-1} \cdots u_{1}=c_{1} \cdots c_{n}$ is a consequence of $P$. Hence

$$
c_{1} \cdots c_{n} u=c_{1} \cdots c_{n}
$$

is a consequence of $P$ and $R$.

Now, assume that $u=v a$, with $a \in X$ and $v \in X^{+}$such that $|v|=n \geq 1$. Then, by the induction hypothesis and the case $n=1$, we have

$$
c_{1} \cdots c_{n} u=\left(c_{1} \cdots c_{n} v\right) a=c_{1} \cdots c_{n} a=c_{1} \cdots c_{n},
$$

as a consequence of $P$ and $R$.

Now, let $k \geq 1$. Since $\delta_{i_{1}, \ldots, i_{k}} \in \mathscr{O}_{n}$, we can take a word $w_{i_{1}, \ldots, i_{k}}$ in $(X \backslash\{g\})^{*}$ that represents $\delta_{i_{1}, \ldots, i_{k}}$. On the other hand, as in $\mathscr{P} \mathscr{O}_{n}$ we have the equality $c_{i_{1}, \ldots, i_{k}}=$ $c_{i_{1}, \ldots, i_{k}} \delta_{i_{1}, \ldots, i_{k}}$, the relation

$$
\prod_{\substack{i=1 \\ i \neq i_{1}, \ldots, i_{k}}}^{n} c_{i}=\left(\prod_{\substack{i=1 \\ i \neq i_{1}, \ldots, i_{k}}}^{n} c_{i}\right) w_{i_{1}, \ldots, i_{k}}
$$

is a consequence of $P$.

Let $\beta$ be the element of $\mathscr{G} \mathscr{P}_{n}$ that is represented by $u$. Then, with $j_{1}=$ $i_{1} \beta, \ldots, j_{k}=i_{k} \beta$, we have $\beta \in \Delta_{j_{1}, \ldots, j_{k}}^{i_{1}, \ldots, i_{k}}$ and $\delta_{i_{i}, \ldots, i_{k}} \beta=\delta_{j_{1}, \ldots, j_{k}}^{i_{1}, \ldots, i_{k}} \in \mathscr{O O P}_{n}$. Hence, since $\langle X \mid R\rangle$ is a presentation for $\mathscr{G P}_{n}$, the relation $w_{i_{1}, \ldots, i_{k}} u=w_{j_{1}, \ldots, j_{k}}^{i_{1}, \ldots, i_{k}}$ is a consequence of $R$.

Thus the relation

$$
w=\left(\prod_{\substack{i=1 \\ i \neq i_{1}, \ldots, i_{k}}}^{n} c_{i}\right) w_{j_{1}, \ldots, j_{k}}^{i_{1}, \ldots, i_{k}}
$$


(whose right-hand side is member of $\bar{W}$ ) is a consequence of $R, P$, and $N$, as required.

Next, we obtain a specific presentation for $\mathscr{P} \mathscr{C} \mathscr{P}{ }_{n}$.

Catarino (1998) showed that $\langle X \mid A, O\rangle$ is a presentation for $\mathscr{G P P}_{n}$, in terms of $2 n-1$ generators and $n^{2}+2 n$ relations, where $A$ is the set of Aizenštat relations on the letters $u_{1}, \ldots, u_{n-1}, v_{1}, \ldots, v_{n-1}$ (see Section 2) and $O$ is the set of the following $2 n$ relations:

$\left(O_{1}\right) g^{n}=1$;

$\left(\mathrm{O}_{2}\right) u_{i} g=g u_{i+1}$, for $1 \leq i \leq n-2$;

$\left(O_{3}\right) v_{i+1} g=g v_{i}$, for $1 \leq i \leq n-2$;

$\left(O_{4}\right) u_{n-1} g=g^{2} v_{n-1} \cdots v_{1}$;

$\left(O_{5}\right) v_{1} g=u_{n-1} \cdots u_{1}$;

$\left(O_{6}\right) g v_{1} \cdots v_{n-1}=v_{1} \cdots v_{n-1}$

Therefore, as a consequence of Proposition 4.7, we have that $\langle Z \mid A, O, P, N\rangle$ is a presentation for $\mathscr{P} \mathscr{G} \mathscr{P}_{n}$, in terms of the $3 n-1$ generators

$$
u_{1}, \ldots, u_{n-1}, v_{1}, \ldots, v_{n-1}, c_{1}, \ldots, c_{n}, g
$$

and $\left(7 n^{2}+5 n-4\right) / 2$ relations: $\left(7 n^{2}-n-4\right) / 2$ relations from $P, 2 n$ relations from $O$ and $n$ relations from $N$. Observe that relations $A$ are contained in relations $P$.

Naturally our next aim is to simplify the presentation $\langle Z \mid R, P, N\rangle$ of $\mathscr{P} \mathscr{P P}_{n}$, using Tietze transformations.

Starting with relations $(N)$ and $\left(O_{1}\right)$, by induction, we obtain the relations $c_{j}=$ $g^{n-j+1} c_{1} g^{j-1}$, for $2 \leq j \leq n$. Similarly, starting with relations $\left(O_{1}\right),\left(O_{2}\right)$ and $\left(O_{3}\right)$, we get the relations $u_{j}=g^{n-j+1} u_{1} g^{j-1}$ and $v_{j}=g^{j-1} v_{1} g^{n-j+1}$, for $2 \leq j \leq n-1$.

Now, using $\left(O_{1}\right)$, the relations $v_{1} g=u_{n-1} \cdots u_{1}$ and $v_{1}=u_{n-1} \cdots u_{1} g^{n-1}$ are equivalent. Then, from relations $u_{j}=g^{n-j+1} u_{1} g^{j-1}$ and $v_{j}=g^{j-1} v_{1} g^{n-j+1}$, for $2 \leq j \leq$ $n-1$, we obtain relations $v_{j}=g^{j}\left(g u_{1}\right)^{n-1} g^{n-j}$, for $1 \leq j \leq n-1$.

Through simple substitutions, we can eliminate relations $\left(A_{1}\right)$ and $\left(A_{2}\right)$ and, from relations $\left(A_{3}\right)$, we obtain the relation

$\left(A_{3}^{\prime}\right)\left(g u_{1}\right)^{n}=g u_{1}$.

We can also eliminate relations $\left(A_{4}\right)$.

With respect to relations $\left(A_{5}\right)$, we can verify that substituting $v_{j}$, for $i=1$ and $1 \leq j \leq n-2$, we obtain the relations $u_{1} g^{j}\left(g u_{1}\right)^{n-1}=g^{j}\left(g u_{1}\right)^{n-1} g^{n-j} u_{1} g^{j}$; for $2 \leq i \leq$ $n-1$ the relations obtained are already included in these last relations. Therefore we can substitute relations $\left(A_{5}\right)$ by the relations

$\left(A_{5}^{\prime}\right) u_{1} g^{j}\left(g u_{1}\right)^{n-1}=g^{j}\left(g u_{1}\right)^{n-1} g^{n-j} u_{1} g^{j}$, for $1 \leq j \leq n-2$.

Relations $\left(A_{6}\right)$ and $\left(A_{7}\right)$ assume the following aspect:

$\left(A_{6}^{\prime}\right)\left(u_{1} g^{n-1} u_{1} g\right) u_{1}=u_{1} g^{n-1} u_{1} g$ 
and

$\left(A_{7}^{\prime}\right) u_{1} g^{2} u_{1}=u_{1} g\left(g u_{1}\right)^{n-2} g^{2} u_{1}$,

respectively.

With respect to relations $\left(E_{1}\right)$, we can verify that substituting $c_{j}$, for $i=1$ and $2 \leq j \leq n$, we obtain the relations $c_{1} g^{n-j+1} c_{1}=g^{n-j+1} c_{1} g^{j-1} c_{1} g^{n-j+1}$; for $2 \leq i \leq n-1$ the relations obtained are already included in these last relations. Therefore we can substitute relations $\left(E_{1}\right)$ by the relations

(E $\left.E_{1}^{\prime}\right) c_{1} g^{n-j+1} c_{1}=g^{n-j+1} c_{1} g^{j-1} c_{1} g^{n-j+1}$, for $2 \leq j \leq n$.

Again, through simple substitutions, we reduce relations $\left(P_{1}\right),\left(P_{2}\right)$, and $\left(P_{3}\right)$, respectively, to

$\left(P_{1}^{\prime}\right) u_{1} c_{1}=u_{1}$,

$\left(P_{2}^{\prime}\right) c_{1} u_{1}=c_{1}$ and

(P) $u_{1}^{\prime} g^{n-1} c_{1}=c_{1} g^{n-1} c_{1}$.

In the case of relations $\left(P_{4}\right)$, we can verify that substituting $c_{i}$ and $u_{j}$, for $j=n-1$ and $1 \leq i \leq n-2$, we obtain the relations $c_{1} g^{i+1} u_{1}=g^{i+1} u_{1} g^{n-i-1} c_{1} g^{i+1}$; for $1 \leq j \leq n-2$ the relations obtained are already included in these last relations. Therefore we can substitute relations $\left(P_{4}\right)$ by the relations

$\left(P_{4}^{*}\right) c_{1} g^{i+1} u_{1}=g^{i+1} u_{1} g^{n-i-1} c_{1} g^{i+1}$, for $1 \leq i \leq n-2$.

Relations $\left(P_{4}^{\prime}\right)$, which assume the aspect $c_{1} g^{n-j} u_{1}=g^{n-j} u_{1} g^{j} c_{1} g^{n-j}$, for $1 \leq j \leq n-2$, are included in $\left(P_{4}^{*}\right)$ and so they can be eliminated.

It is easy to verify that relations $\left(P_{5}\right)$ and $\left(P_{6}\right)$ can also be eliminated and relations $\left(P_{7}\right)$ can be reduced to the relation

$\left(P_{7}^{\prime}\right)\left(g u_{1}\right)^{n-1} g c_{1}=c_{1} g c_{1}$.

A reasoning similar to that used for $\left(P_{4}\right)$, allows us to conclude that relations $\left(P_{8}\right)$ can be reduced to

$\left(P_{8}^{*}\right) c_{1} g^{i}\left(g u_{1}\right)^{n-1}=g^{i}\left(g u_{1}\right)^{n-1} g^{n-i} c_{1} g^{i}$, for $1 \leq i \leq n-2$.

Relations $\left(P_{8}^{\prime}\right)$ take the form

$$
c_{1} g^{n-j-1}\left(g u_{1}\right)^{n-1}=g^{n-j-1}\left(g u_{1}\right)^{n-1} g^{j+1} c_{1} g^{n-j-1} \text {, for } 1 \leq j \leq n-2,
$$

which are included in $\left(P_{8}^{*}\right)$, and so they can also be eliminated.

Starting with relations $\left(O_{1}\right), v_{j}=g^{j}\left(g u_{1}\right)^{n-1} g^{n-j}$, for $1 \leq j \leq n-1$, and $\left(A_{3}^{\prime}\right)$ we can eliminate relation $\left(O_{4}\right)$.

From relations $v_{j}=g^{j}\left(g u_{1}\right)^{n-1} g^{n-j}$, for $1 \leq j \leq n-1$, we obtain the relation $\left(O_{6}^{\prime}\right) g\left[g\left(g u_{1}\right)^{n-1}\right]^{n-1}=\left[g\left(g u_{1}\right)^{n-1}\right]^{n-1}$.

Therefore we have showed that, applying elementary Tietze transformations, letters $u_{2}, \ldots, u_{n-1}, c_{2}, \ldots, c_{n}, v_{1}, \ldots, v_{n-1}$ can be eliminated and so we obtain a presentation for $\mathscr{P} \mathscr{O P}{ }_{n}$ in terms of three generators $\left(u=u_{1}, c=c_{1}\right.$, and $\left.g\right)$ and 
the following set $Q$ of $4 n+2$ relations:

$\left(A_{3}^{\prime}\right)(g u)^{n}=g u$;

$\left(A_{5}^{\prime}\right) u g^{j}(g u)^{n-1}=g^{j}(g u)^{n-1} g^{n-j} u g^{j}$, for $1 \leq j \leq n-2$;

$\left(A_{6}^{\prime}\right)\left(u g^{n-1} u g\right) u_{1}=u g^{n-1} u g$;

$\left(A_{7}^{\prime}\right) u g^{2} u=u g(g u)^{n-2} g^{2} u$;

$\left(E_{1}^{\prime}\right) c g^{n-j+1} c=g^{n-j+1} c g^{j-1} c g^{n-j+1}$, for $2 \leq j \leq n$

$\left(P_{1}^{\prime}\right) u c=u$

$\left(P_{2}^{\prime}\right) c u=c$

$\left(P_{3}^{\prime}\right) u g^{n-1} c=c g^{n-1} c$;

( $\left.P_{4}^{*}\right) c g^{i+1} u=g^{i+1} u g^{n-i-1} c g^{i+1}$, for $1 \leq i \leq n-2$;

$\left(P_{7}^{\prime}\right)(g u)^{n-1} g c=c g c$;

$\left(P_{8}^{*}\right) c g^{i}(g u)^{n-1}=g^{i}(g u)^{n-1} g^{n-i} c g^{i}$, for $1 \leq i \leq n-2$;

$\left(O_{1}\right) g^{n}=1$;

$\left(O_{6}^{\prime}\right) g\left[g(g u)^{n-1}\right]^{n-1}=\left[g(g u)^{n-1}\right]^{n-1}$.

Theorem 4.8. The monoid $\mathscr{P} \mathscr{O P}_{n}$ admits the presentation $\langle u, g, c \mid Q\rangle$ with three generators and $4 n+2$ relations.

\section{A PRESENTATION FOR $\mathscr{P} \mathscr{G} \mathscr{R}_{n}$}

In this last section we look at the monoid $\mathscr{P} \mathscr{G} \mathscr{R}_{n}$ of all orientation preserving or reversing partial transformations on $X_{n}$. We obtain a presentation for $\mathscr{P} \mathscr{G} \mathscr{R}_{n}$ using the same technique applied in Section 3.

Let $\langle u, g, c \mid Q\rangle$ be the presentation for $\mathscr{P} \mathscr{C} \mathscr{P}_{n}$ given in Theorem 4.8. Again, an argument similar to the one used to prove Proposition 2.1 allows us to conclude the following.

Proposition 5.1. The set $\bar{Z}=\{u, g, c, h\}$ generates $\mathscr{P} \mathscr{G} \mathscr{R}_{n}$.

Consider the following set of relations:

$\left(M_{1}\right) h u=g(g u)^{n-1} g^{n-1} h$;

$\left(M_{2}\right) h g=g^{n-1} h$;

$\left(M_{3}\right) h c=g c g^{n-1} h$;

$\left(M_{4}\right)(g c)^{n-2} g^{2} h=(g c)^{n-2} g\left(u g^{n-1}\right)^{n-3} u g^{n-3}$;

$\left(M_{5}\right) h^{2}=1$.

It is a routine matter to prove that the above relations over the alphabet $\bar{Z}$ are satisfied by the generating set $\bar{Z}$ of $\mathscr{P} \mathscr{O} \mathscr{R}_{n}$.

Define $\bar{R}=Q \cup M_{1} \cup M_{2} \cup M_{3} \cup M_{4} \cup M_{5}$ and observe that $|\bar{R}|=4 n+7$.

Take a set of forms $W^{\prime}$ for $\mathscr{P} \mathscr{O P}{ }_{n}$ associated to the presentation $\langle\bar{Z} \backslash\{h\} \mid Q\rangle$, containing the empty word (for technical reasons).

Let $u_{0}=(g c)^{n-2} g^{2}=\left(\begin{array}{ll}1 & 2 \\ 1 & 2\end{array}\right)$.

Let $a$ be an element of $\mathscr{P} \mathscr{G} \mathscr{P}_{n}$ with rank one or two. If $\operatorname{Im}(a)=\{i, j\}$ and $\operatorname{Dom}(a)=\left\{i_{1}<\cdots<i_{s}\right\}$, then

$$
a=\left(\begin{array}{ccc|ccc|ccc}
i_{1} & \cdots & i_{k} & i_{k+1} & \cdots & i_{k+l} & i_{k+l+1} & \cdots & i_{s} \\
i & \cdots & i & j & \cdots & j & i & \cdots & i
\end{array}\right),
$$


for some $0 \leq k, l \leq s$, and $s \geq 1$. Take the elements of $\mathscr{P} \mathscr{O} \mathscr{P}{ }_{n}$

$$
\alpha_{a}=\left(\begin{array}{ccc|ccc|ccc}
i_{1} & \cdots & i_{k} & i_{k+1} & \cdots & i_{k+l} & i_{k+l+1} & \cdots & i_{s} \\
1 & \cdots & 1 & 2 & \cdots & 2 & 1 & \cdots & 1
\end{array}\right) \quad \text { and } \quad \beta_{a}=\left(\begin{array}{ll}
1 & 2 \\
i & j
\end{array}\right)
$$

It is obvious that $a=\alpha_{a} u_{0} \beta_{a}$.

Let $u_{a}, v_{a} \in W^{\prime}$ be the words that represents $\alpha_{a}$ and $\beta_{a}$, respectively. Observe that the word $u_{a} u_{0} v_{a}$ also represents $a$. Moreover, if $v_{0} \in W^{\prime}$ is the word that represents the empty transformation, then the word $u_{0} v_{0}$ also represents the empty transformation.

Next, let $W_{\alpha}=\left\{u_{a} u_{0} v_{a}: a \in J_{1} \cup J_{2}\right\} \cup\left\{u_{0} v_{0}\right\}$ and $W_{\beta}=\left\{w \in W^{\prime}: w \varphi \in J_{3} \cup\right.$ $\left.\cdots \cup J_{n}\right\}$, where $\varphi$ is the canonical morphism from $(\bar{Z} \backslash\{h\})^{*}$ onto $\mathscr{P} \mathscr{C P}_{n}$. Then $W=$ $W_{\alpha} \cup W_{\beta}$ is a new set of forms for $\mathscr{P} \mathscr{G} \mathscr{P}_{n}$, where $W_{\alpha}$ is the set of forms that represent the transformations of $\mathscr{P} \mathscr{O} \mathscr{P}_{n}$ of rank less than or equal two.

Consider $\bar{W}=W \cup\left\{w h: w \in W_{\beta}\right\}$. Notice that

$$
|\bar{W}|=2\left|\mathscr{P} \mathscr{P}_{n}\right|-\left[2\left(\begin{array}{l}
n \\
2
\end{array}\right)^{2} 2^{n-2}+\left(2^{n}-1\right) n+1\right]=\left|\mathscr{P} \mathscr{R} \mathscr{R}_{n}\right|
$$

and $u_{0}$ is a factor of each word in $W_{\alpha}$. Hence, by Theorem 1.3, we have the following result:

Theorem 5.2. The monoid $\mathscr{P O C}_{n}$ is defined by the presentation $\langle\bar{Z} \mid \bar{R}\rangle$, on 4 generators and $4 n+7$ relations.

\section{ACKNOWLEDGMENTS}

This work was developed within the activities of Centro de Álgebra da Universidade de Lisboa, supported by FCT and FEDER, within project POCTI "Fundamental and Applied Algebra" and, for the first author, it was also prepared within the project JD: "Apresentações para semigrupos", FCT-UNL, 1999.

\section{REFERENCES}

Aǐzenštat, A. Ya. (1962). The defining relations of the endomorphism semigroup of a finite linearly ordered set. Sibirsk. Mat. 3:161-169 (in Russian).

Arthur, R. E., Ruškuc, N. (2000). Presentations for two extensions of the monoid of orderpreserving mappings on a finite chain. Southeast Asian Bull. Math. 24:1-7.

Catarino, P. M. (1998). Monoids of orientation-preserving transformations of a finite chain and their presentations. Semigroups and Applications. J. M. Howie and N. Ruškuc, eds., World Scientific: $39-46$.

Catarino, P. M., Higgins, P. M. (1999). The monoid of orientation-preserving mappings on a chain. Semigroup Forum 58:190-206.

Cowan, D. F., Reilly, N. R. (1995). Partial cross-sections of symmetric inverse semigroups. Int. J. Algebra Comput. 5:259-287.

Delgado, M., Fernandes, V. H. (2000). Abelian kernels of some monoids of injective partial transformations and an application. Semigroup Forum 61:435-452.

Delgado, M., Fernandes, V. H. (2004). Abelian kernels of monoids of order-preserving maps and of some of its extensions. Semigroup Forum 68:335-356. 
Fernandes, V. H. (1997). Semigroups of order-preserving mappings on a finite chain: a new class of divisors. Semigroup Forum 54:230-236.

Fernandes, V. H. (1998). Normally ordered inverse semigroups. Semigroup Forum 58: $418-433$.

Fernandes, V. H. (2000). The monoid of all injective orientation preserving partial transformations on a finite chain. Comm. Alg. 28:3401-3426.

Fernandes, V. H. (2001). The monoid of all injective order preserving partial transformations on a finite chain. Semigroup Forum 62:178-204.

Fernandes, V. H. (2001). A division theorem for the pseudovariety generated by semigroups of orientation preserving transformations on a finite chain. Comm. Alg. 29:451-456.

Fernandes, V. H. (2002a). Presentations for some monoids of partial transformations on a finite chain: a survey. Semigroups, Algorithms, Automata and Languages (Gracinda M. S. Gomes, Jean-Éric Pin, Pedro V. Silva, eds.). World Scientific, pp. 363-378.

Fernandes, V. H. (2002b). Semigroups of order-preserving mappings on a finite chain: another class of divisors. Izvestiya VUZ. Matematika 3(478):51-59 (in Russian).

Fernandes, V. H., Gomes, G. M. S., Jesus, M. M. (to appear). Presentations for some monoids of injective partial transformations on a finite chain. Southeast Asian Bull. Math.

The GAP Group. GAP-Groups, Algorithms, and Programming, Version 4.3, 2002. (http://www.gap-system.org).

Gomes, G. M. S., Howie, J. M. (1992). On the ranks of certain semigroups of orderpreserving transformations. Semigroup Forum 45:272-282.

Howie, J. M. (1971). Product of idempotents in certain semigroups of transformations. Proc. Edinburgh Math. Soc. 17:223-236.

Lallement, G. (1979). Semigroups and Combinatorial Applications. John Wiley \& Sons, 1979.

McAlister, D. (1997). Semigroup for Windows. Northern Illinois University.

McAlister, D. (1998). Semigroups generated by a group and an idempotent. Comm. Alg. 26:515-547.

Popova, L. M. (1962). The defining relations of the semigroup of partial endomorphisms of a finite linearly ordered set. Leningradskij gosudarstvennyj pedagogicheskij institut imeni A. I. Gerzena. Uchenye Zapiski 238:78-88 (in Russian).

Ruškuc, N. (1995). Semigroup presentations. Ph.D. thesis, University of St-Andrews. 